\title{
Tumours mimicking sports injury in two young athletes
}

\author{
N. Maffulli ${ }^{1}$, MD, E. Pintore ${ }^{2}$, MD and F. Petricciuolo ${ }^{2}$, MD \\ ${ }^{1}$ Hospital for Sick Children, Department of Orthopaedics, Great Ormond Street, London and Institute of Child \\ Health, Sports Medicine Laboratory, London, UK \\ ${ }^{2}$ Departement d'Orthopedie et Traumatologie, Centre Hospitalier d'Annecy, Annecy, France
}

We report two athletic youngsters who had originally been treated for sports injuries around the shoulder joint, and who were eventually discovered to be suffering from bone tumours.

\section{Case 1}

A nine year old male swimmer was referred with a two month history of left shoulder pain. During a pre-championship intensive training period, he developed left shoulder pain, worse after training which made him curtail his training schedule. His GP had diagnosed subacromial impingement of the supraspinatus and of the biceps tendons. Two months' treatment with modified rest, physiotherapy, ice packs and ultrasound were of no benefit.

On examination, the left shoulder was slightly tender over the lateral end of the spine of the scapula. Range of motion was limited to $100^{\circ}$ in abduction and about $120^{\circ}$ in flexion. Neurological examination was normal. X-ray showed a multifocal osteolytic area, extending from the neck of the scapula to the glenoid cavity (Figure 1). A CT scan showed erosion of the anterior and posterior cortex of the scapula (Figure 2).

The tumour was resected $1 \mathrm{~cm}$ medially to its medial margin through a posterior approach. No evidence of soft tissue involement was found. Histology showed a giant cell tumour.

Mobilization was started the day after operation. In the six months since surgery, the patient has remained well. Movement is limited to about $90^{\circ}$ in abduction and $110^{\circ}$ in flexion. The shoulder is stable, despite post-operative retroversion of the pseudojoint (Figure 3) and the inevitable ligamentous damage. He has not been able to resume competitive swimming, but is leading an otherwise normal life.

\section{Case 2}

A sixteen year old male soccer player was referred for a second opinion. He gave a four month history of painful right shoulder following a tackle with an

Address for Correspondence: Dr N. Maffulli, Institute of Child Health, Sports Medicine Laboratory, 30 Guildford Street, London WC1, UK

(C) 1990 Butterworth-Heinemann Ltd 0306-4179/90/030207-02



Figure 1. X-ray taken two months after the onset of pain. A multilocular osteolytic area extending laterally from the scapular neck to the glenoid cavity is seen

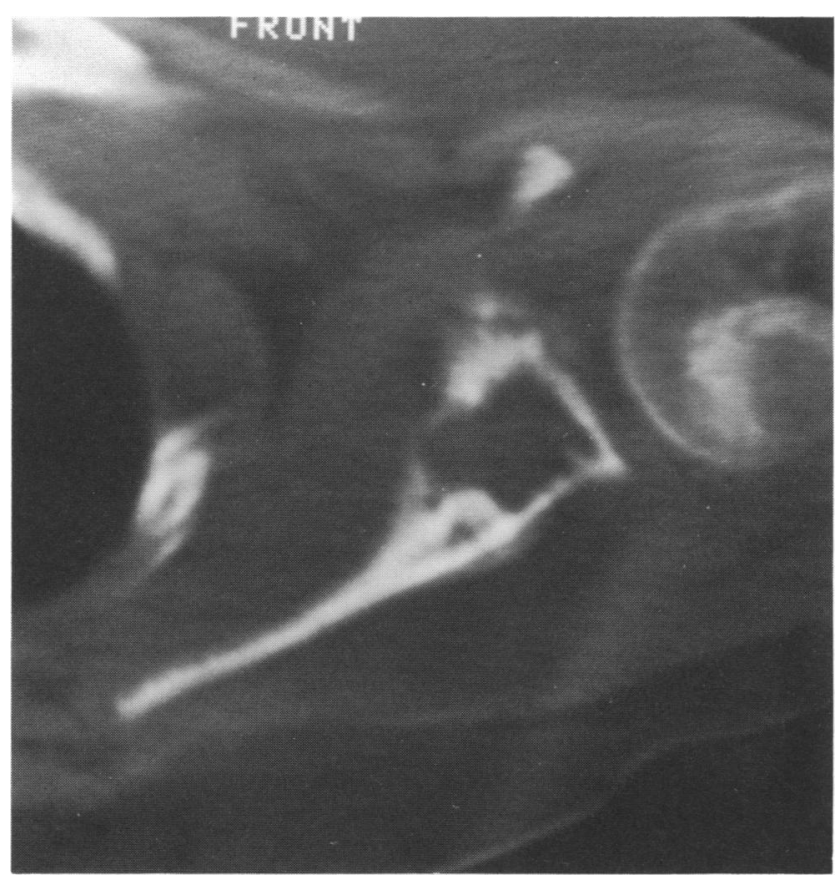

Figure 2. CT scan in the horizontal plane showing erosion of the anterior and posterior cortex 


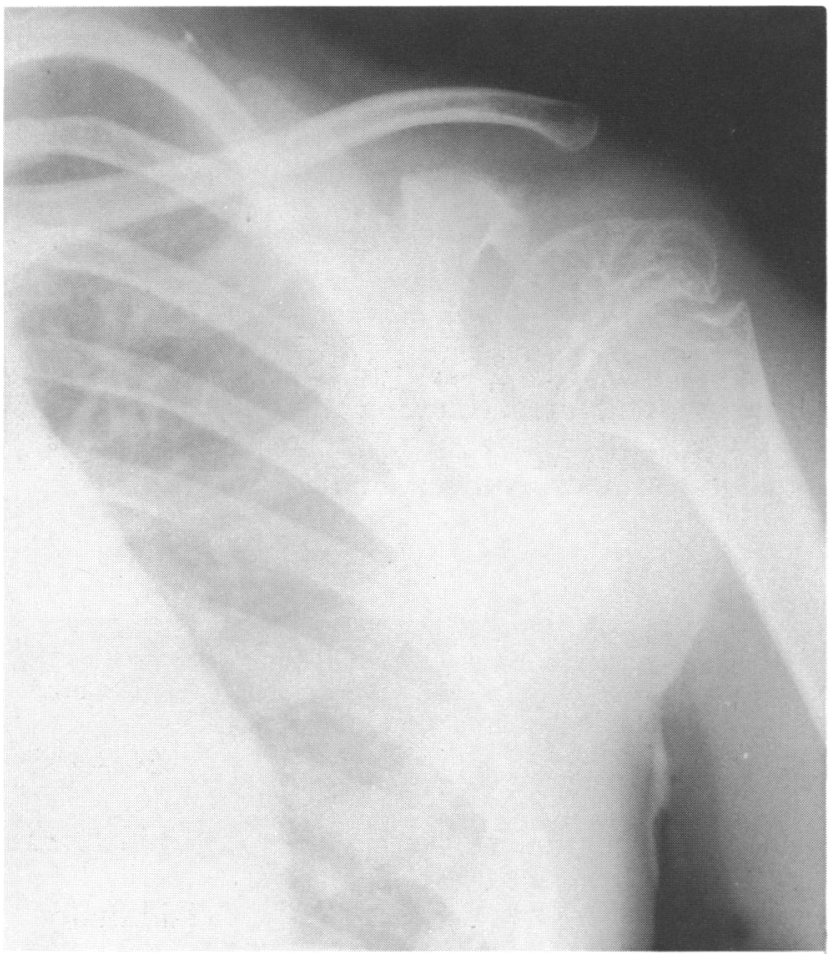

Figure 3. X-ray taken six months after operation. No recurrence is evident. A pseudo-joint is seen

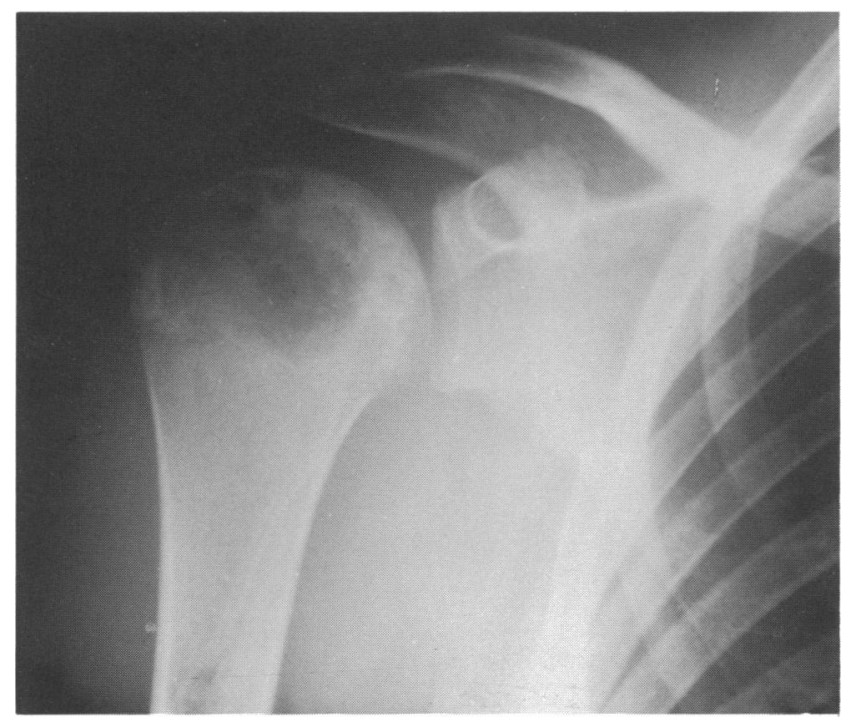

Figure 4. Pre-operative $X$-ray showing a lytic lesion of the whole of the humeral head

opponent during a match. The pain was worse during the night. A soft tissue injury had been diagnosed by another orthopaedic surgeon. The patient had been treated intermittently for four months with physiotherapy, ultrasound and nonsteroidal anti-inflammatory drugs. The pain was better during the treatment periods, but never disappeared completely.

On examination, there was localized tenderness over the anterior aspect of the right shoulder, radiating to the rotator cuff. Range of motion was

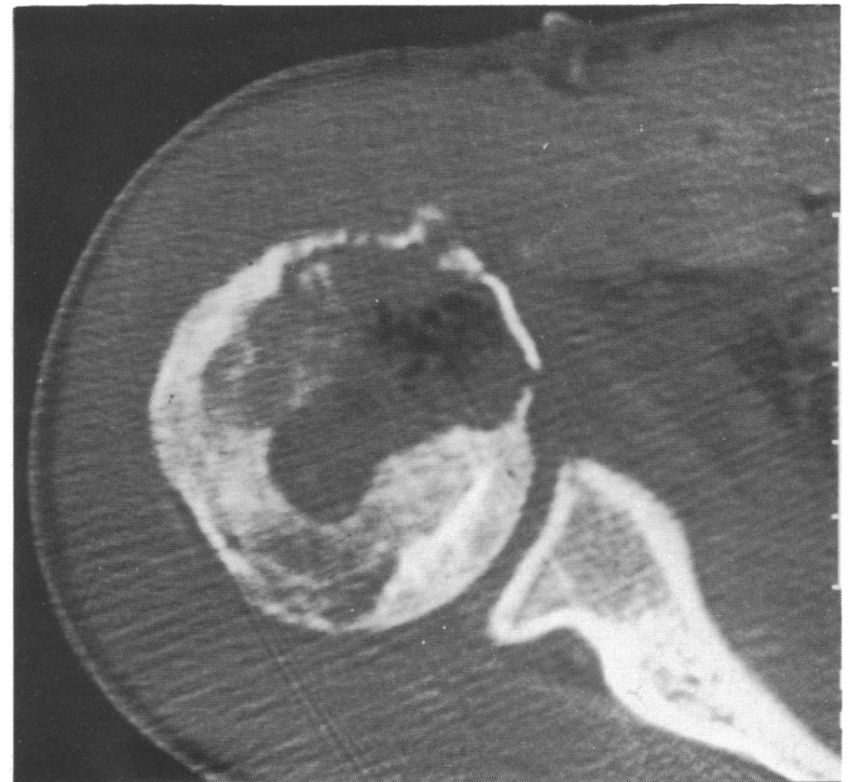

Figure 5. CT scan showing the extent of the above lesion and thinning of the cortex

limited to $80^{\circ}$ in abduction and external rotation. Neurological examination was unremarkable. X-ray showed a multifocal osteolytic area in the humeral head (Figure 4). A CT scan confirmed the extent of the lesion, and showed a thinned cortex (Figure 5).

The patient underwent surgery through a deltopectoral approach. The tumour cavity was curetted through a $2 \mathrm{~cm}$ in diameter anterior opening, and washed out with saline. Three different laboratories diagnosed a benign epiphyseal chondroblastoma.

Mobilization was started the day after operation. Six months after surgery, the patient is well with no evidence of recurrence. He has regained full movement and resumed soccer.

\section{Discussion}

Only about one percent of 1350 giant cell tumours in a recent series were located in the scapula ${ }^{1}$. About a quarter were in patients under ten and the majority in patients under 20 years. Benign epiphyseal chondroblastoma generally involves the epiphysis of long or short bones, the humeral head being a common site $^{2}$. Neither of these tumours is fatal, but giant cell tumours may recur locally'.

If an apparent sports injury does not improve after an appropriate period, then more serious causes must be sought ${ }^{3}$.

\section{References}

1 Aoiki, J., Moster, R.P. and Vinh, T.N. Giant cell tumor of the scapula. A review of 13 cases Skeletal Radiol 1989, 18, 427-434

2 Schajowicz, F. and Santini Araujo, E.H. Cysts and tumours of the musculoskeletal system-pathology. In 'Postgraduate Textbook of Clinical Orthopaedics' N.H. Harris (Ed.) Bristol, Wright, 1983, 605-639

3 Lewis, M.M. and Reilly, J.F. Sports tumors Am J Sports Med 1987, 15, 362-365 